\title{
Analgesia Perioperatória com Infusão Peridural Contínua da Combinação de Morfina e Clonidina em Crianças Submetidas a Procedimentos Cirúrgicos Abdominais*
} Perioperative Analgesia with Continuous Epidural Infusion of Morphine Combined with Clonidine in Children Undergoing Abdominal Surgeries

\author{
Jyrson Guilherme Klamt, TSA ${ }^{1}$, Magaly Santoni ${ }^{2}$, Luis Vicente Garcia, TSA ${ }^{1}$, Renato Mestriner Stocche, TSA ${ }^{3}$
}

\section{RESUMO}

Klamt JG; Santoni M, Garcia LV, Stocche RM — Analgesia Perioperatória com Infusão Peridural Contínua da Combinação de Morfina e Clonidina em Crianças Submetidas a Procedimentos Cirúrgicos Abdominais.

JUSTIFICATIVA E OBJETIVOS: O presente estudo foi desenvolvido para avaliar o efeito analgésico da combinação de morfina e clonidina administrada por via peridural, cuja eficácia foi demonstrada em pacientes adultos, sobre o consumo de isoflurano e o consumo de medicação analgésica no período pós-operatório em crianças.

MÉTODO: Vinte e seis crianças escaladas para operações intraabdominais foram alocadas de forma aleatória em dois grupos. Os dois grupos receberam, por via peridural, bolus de morfina $\left(8 \mu \mathrm{g} \cdot \mathrm{kg}^{-1}\right)$ e de clonidina $\left(0,8 \mu \mathrm{g} \cdot \mathrm{kg}^{-1}\right)$ antes do início da intervenção cirúrgica, seguidos de infusão contínua de clonidina $(0,12$ $\left.\mu \mathrm{g} \cdot \mathrm{kg}^{-1} \cdot \mathrm{h}^{-1}\right)$ mais morfina $\left(1,2 \mu \mathrm{g} \cdot \mathrm{kg}^{-1} \cdot \mathrm{h}^{-1}\right)$ no Grupo I e o dobro dessas doses no Grupo II, durante 24 horas. Foram medidas as concentrações inspiratórias de isoflurano durante a operação e 0 número de doses (1 mg. $\left.\mathrm{kg}^{-1}\right)$ de tramadol durante 24 horas no pósoperatório.

RESULTADOS: As concentrações de isoflurano foram significativamente menores em relação aos valores observados antes da incisão cirúrgica após 60 e 90 minutos nos Grupos II e I, respectivamente, porém não houve diferença entre os dois grupos. O con-

\footnotetext{
*Recebido do (Received from) Serviço de Anestesiologia do Hospital das Clínicas de Ribeirão Preto da Faculdade de Medicina de Ribeirão Preto - Universidade de São Paulo (HCFMRP-USP)

1. Professor-Asssistente-Doutor da Disciplina de Anestesiologia do Departamento de Biomecânica, Medicina e Reabilitação do Aparelho Locomotor, FMRP. USP

2. Médica Assistente, Hospital São Vicente de Paulo (Jundiaí-SP); Doutor em Ciências Médicas pela FMRP-USP

3. Médico Assistente do Serviço de Anestesiologia do HCFMRP-USP; Doutor em Ciências Médicas pela FMRP-USP
}

Apresentado (Submitted) em 22 de maio de 2006

Aceito (Accepted) para publicação em 06 de agosto de 2007

Endereço para correspondência (Correspondence to):

Dr. Jyrson Guilherme Klamt

Serviço de Anestesiologia - Hospital das Clínicas

Av. Bandeirantes 3.900 - Monte Alegre

14048-900 Ribeirão Preto, SP

E-mail: jgklamt@fmrp.usp.br

(C) Sociedade Brasileira de Anestesiologia, 2007 sumo de tramadol foi muito menor no Grupo II, e sete (53,8\%) não necessitaram analgesia de resgate, porém foram observadas maior sedação e hipotensão arterial. Não foi observada depressão respiratória nos dois grupos.

CONCLUSÕES: A infusão peridural da combinação de baixas doses de clonidina e morfina promoveu redução da necessidade de isoflurano no período intra-operatório e analgesia pós-operatória de boa qualidade.

Unitermos: ANALGESIA, Regional: espinal; ANALGÉSICOS: morfina, clonidina; CIRURGIA, Pediátrica.

\section{SUMMARY}

Klamt JG, Santoni M, Garcia LV, Stocche RM - Perioperative Analgesia with Continuous Epidural Infusion of Morphine Combined with Clonidine in Children Undergoing Abdominal Surgeries.

BACKGROUND AND OBJECTIVES: The present study was developed to evaluate the analgesic effects of the epidural administration of a combination of morphine and clonidine, whose efficacy has been demonstrated in adults, on the consumption of isoflurane and postoperative consumption of analgesics in children.

METHODS: Twenty-six children scheduled for intra-abdominal surgeries were randomly divided in two groups. Both groups received an epidural bolus of morphine $\left(8 \mu \mathrm{g} \cdot \mathrm{kg}^{-1}\right)$ and clonidine $\left(0.8 \mu \mathrm{g} . \mathrm{kg}^{-1}\right)$ before the surgery, followed by the continuous infusion of clonidine $\left(0.12 \mu \mathrm{g} \cdot \mathrm{kg}^{-1} \cdot \mathrm{h}^{-1}\right)$ plus morphine $\left(1.2 \mu \mathrm{g} \cdot \mathrm{kg}^{-1} \cdot \mathrm{h}^{-1}\right)$ in Group I, and twice those dosages in Group II, during 24 hours. Inspiratory concentrations of isoflurane were measured during the surgery, as well as the number of doses $\left(1 \mathrm{mg} \cdot \mathrm{kg}^{-1}\right)$ of tramadol during 24 hours in the postoperative period.

RESULTS: The concentrations of isoflurane were significantly smaller after 60 and 90 minutes than the concentrations before the incision in Groups II and I, respectively, but those differences were not statistically significant. The consumption of tramadol was significantly lower in Group II, and 7 (53.8\%) patients in this group did not need rescue analgesia, but we observed an increase in the incidence of sedation and hypotension. Respiratory depression was not observed in either group.

CONCLUSIONS: Epidural infusion of low doses of clonidine combined with morphine reduced the intraoperative need of isoflurane and postoperative analgesia

Key Words: ANALGESIA, Regional: spinal; ANALGESICS: morphine, clonidine; SURGERY, pediatrics. 


\section{INTRODUÇÃO}

A administração peridural de clonidina, um agonista $\alpha_{2}$-adrenérgico, produz analgesia ${ }^{1}$ e prolonga a analgesia produzida pela ropivacaína por via peridural caudal em crianças ${ }^{2}$. Devido à duração limitada do efeito após administração em bolus, faz-se necessária a infusão contínua para prolongar a analgesia no período pós-operatório. Mesmo nos adultos, há poucos dados na literatura sobre regimes de doses da infusão peridural de clonidina ${ }^{3,4}$. Recentemente, dois estudos confirmaram, em crianças, a segurança e a eficácia da infusão peridural de clonidina associada a doses pequenas de bupivacaína ${ }^{5,6}$. Um estudo preliminar ${ }^{7}$ demonstrou que a administração peridural, em bolus, de clonidina de forma isolada ou associada a ropivacaína seguida de infusão peridural dos mesmos fármacos promove analgesia de boa qualidade contra a dor no repouso em crianças que se submetem a intervenção cirúrgica abdominal intracavitária. No entanto, a segurança da infusão peridural de clonidina é limitada pelos seus efeitos sedativos e de hipotensão arterial ${ }^{7}$.

Vários estudos realizados em pacientes adultos demonstraram a potente interação da administração peridural concomitante de morfina e clonidina no alívio da dor pós-operatória em procedimentos cirúrgicos abdominais ${ }^{8-12}$.

A utilização de doses pequenas de morfina e clonidina permite reduzir, de modo significativo, o risco potencial de graves efeitos adversos da morfina peridural (depressão respiratória, retenção urinária, vômitos, prurido e sedação) e da clonidina peridural (sedação e hipotensão arterial). Além do mais, a depressão respiratória tardia, efeito adverso mais temido após administração peridural de morfina, não é intensificada pela clonidina. A curta duração de ação de ambas os fármacos tornam a infusão contínua mais adequada para o alívio prolongado da dor após intervenções cirúrgicas de grande porte quando comparada com injeções intermitentes ${ }^{13}$. A despeito da comprovada eficácia da combinação de morfina com clonidina nos pacientes adultos, nenhum estudo prévio foi conduzido para avaliar regimes de administração peridural contínua da combinação de morfina com clonidina no controle da dor em pacientes pediátricos. Some-se a isso, a possibilidade de que a redução da necessidade de anestésicos gerais no intra-operatório pode ser um benefício adicional resultante da combinação da morfina e clonidina por via peridural ${ }^{1,12,14-16}$. Assim, o presente estudo avalia a eficácia da infusão peridural de baixas doses de morfina e clonidina em crianças submetidas a procedimentos cirúrgicos abdominais de grande porte.

\section{MÉTODO}

Após aprovação pelo Comitê de Ética em Pesquisa do Hospital das Clínicas da Faculdade de Medicina de Ribeirão Preto (USP) e obtenção do consentimento informado por escrito dos pais ou responsáveis legais, foram recrutadas 26 crianças, com idades entre 4 e 48 meses, estados físi- co ASA I a III escaladas para intervenções cirúrgicas abdominais de grande porte, tais como fundoplicatura a Nissen, excisão de tumores intra-abdominais, esplenectomia ou cirurgias urológicas intracavitárias. Os pacientes foram eletronicamente alocados de modo aleatório (Microsoft Excel, Windows) em dois grupos de tratamento, não encobertos, de analgesia pós-operatória. Os dois grupos receberam infusão contínua da mistura da clonidina $\left(1,2 \mu \mathrm{g} \cdot \mathrm{mL}^{-1}\right)$ e morfina $\left(12 \mu \mathrm{g} \cdot \mathrm{mL}^{-1}\right)$ em solução fisiológica a $0,9 \%$ durante 24 horas por meio de cateter peridural, precedida de um bolus de clonidina $\left(0,8 \mu \mathrm{g} \cdot \mathrm{kg}^{-1}\right)$ e morfina $\left(8 \mu \mathrm{g} \cdot \mathrm{kg}^{-1}\right)$ cerca de cinco minutos antes da incisão cirúrgica. Os ritmos de infusões foram de 0,1 e $0,2 \mathrm{~mL} \cdot \mathrm{kg}^{-1} \cdot \mathrm{h}^{-1}$ para os grupos I e II, respectivamente. Os critérios de exclusão foram a recusa dos pais ou responsáveis pela anestesia peridural, doença neurológica, doença cardiovascular ou instabilidade hemodinâmica. As crianças foram colocadas em decúbito lateral e um cateter peridural (20G, Becton-Dickinson) foi inserido por via lombar $\left(L_{1}-L_{5}\right)$ ou caudal (hiato sacro) através de agulha tipo Touhy (18G, Becton-Dickinson) cerca de $5 \mathrm{~cm}$ no espaço peridural. Apenas as crianças nas quais o cateter pôde ser introduzido com facilidade e ofereceu resistência normal à injeção foram incluídas no estudo.

A anestesia geral foi induzida com midazolan $\left(0,1 \mathrm{mg} \mathrm{kg}^{-1}\right)$, lidocaína (1,5 mg.kg-1), propofol (3-5 mg. $\left.\mathrm{kg}^{-1}\right)$, fentanil $\left(5 \mu \mathrm{g} \cdot \mathrm{kg}^{-1}\right)$, dexametasona (1 mg.kg-1), ondansentrona $\left(0,2 \mathrm{mg} \cdot \mathrm{kg}^{-1}\right)$ e pancurônio $\left(0,15 \mathrm{mg} \cdot \mathrm{kg}^{-1}\right)$, e mantida com $\mathrm{N}_{2} \mathrm{O}\left(\mathrm{N}_{2} \mathrm{O} / \mathrm{O}_{2}, 1: 1\right)$ $\mathrm{e}$ isoflurano. A concentração inspiratória de isoflurano foi titulada para manter a estabilidade hemodinâmica, definida como uma variação da pressão arterial sistólica e freqüência cardíaca de, no máximo, de $20 \%$ dos valores antes da indução. As concentrações inspiratórias de isoflurano foram registradas imediatamente antes da incisão cirúrgica, 10 , 20, 30, 40, 50, 60 e 90 minutos após a incisão e durante os fechamentos da aponeurose e da pele. Durante o procedimento cirúrgico, as crianças receberam, para hidratação, por via venosa, solução de Ringer com lactato (12-15 mL.kg.h $\left.{ }^{-1}\right)$ e solução glicosada ( $\left.5 \mathrm{mg} \cdot \mathrm{kg}^{-1} \cdot \mathrm{mm}^{-1}\right)$. Uma sonda orogástrica foi colocada durante a intervenção cirúrgica para drenagem gástrica contínua.

Após o fechamento da parede abdominal, o bloqueio neuromuscular residual foi revertido com atropina $\left(25 \mu \mathrm{g} . \mathrm{kg}^{-1}\right) \mathrm{e}$ neostigmina $\left(50 \mu \mathrm{g} \cdot \mathrm{kg}^{-1}\right)$ e permitida a ventilação espontânea se os pacientes pudessem manter a $\mathrm{SpO}_{2} \geq 95 \%$ e a $\mathrm{P}_{\mathrm{ET}} \mathrm{CO}_{2} \leq 55 \mathrm{mmHg}$. O tempo decorrido entre o fim da intervenção cirúrgica e o início da ventilação espontânea e o tempo durante $o$ qual a anestesia geral foi mantida somente com $\mathrm{N}_{2} \mathrm{O}$ foram registrados em minutos. A extubação foi realizada quando a criança reagiu fortemente à cânula traqueal, exibiu freqüência e padrão de ventilação espontânea normais, teve $\mathrm{SpO}_{2} \geq 97 \%\left(\mathrm{FiO}_{2}=1,0\right.$ e $\left.\mathrm{P}_{\mathrm{ET}} \mathrm{CO} 2<45 \mathrm{mmHg}\right)$, abriu os olhos e movimentou os quatro membros. Após o procedimento cirúrgico, as crianças foram transportadas para a sala de recuperação pós-anestésica (SRPA) ou para Unidade de Terapia Intensiva Pediátrica (UTIP) (pacientes 
com menos de 6 meses de vida) quando puderam manter $\mathrm{SpO}_{2} \geq 95 \%$ respirando ar ambiente por cinco minutos e atingiram escores de recuperação (escala de Aldrete-Kroulik modificada ${ }^{14}$ ) $\geq 7$ e sedação (escala de $0-5$ ) $\geq 3$. O tempo necessário para extubação e para a transferência para SRPA ou UTIP também foi registrado.

A equipe de enfermagem foi orientada para administrar tramadol ( $1 \mathrm{mg} \cdot \mathrm{kg}^{-1}$, no máximo $3 \mathrm{mg} \cdot \mathrm{kg}^{-1} \mathrm{em}$ um período de seis horas) por via venosa (analgesia de resgate) quando fosse identificado dor/desconforto nos pacientes: choro e agitação não consolável, queixa de dor, dificuldade para tossir e mobilização no leito devido à dor, ou pressão arterial sistólica ou freqüência cardíaca superior a $20-30 \%$ dos valores pré-operatórios. Soluções de tramadol $\left(1 \mathrm{mg} \cdot \mathrm{mL}^{-1}\right)$ estiveram sempre disponíveis para administração imediata, as quais eram prescritas pela equipe de anestesia e administradas segundo avaliação da enfermeira responsável pela criança. Os pais ou responsáveis foram esclarecidos a respeito dos possíveis sinais de dor/desconforto e estimulados a comunicar a equipe de enfermagem se julgassem que sua criança estivesse com dor e/ou desconforto. A qualidade da analgesia pós-operatória foi avaliada pelo número de doses de tramadol solicitadas durante 24 horas no período pós-operatório e considerado o segundo parâmetro de eficácia do estudo. Nenhuma paciente envolvida no presente estudo recebeu morfina e/ou dipirona durante 24 horas.

Sedação, pressão arterial (PA), freqüência cardíaca (FC), recuperação da anestesia (escala de Aldrete-Kroulik modificada) e freqüência respiratória (FR) foram registradas a intervalos de 30 minutos na SRPA e na UTIP até 120 minutos. As crianças que permaneceram na SRPA receberam alta para enfermaria após 120 minutos de suas chegadas nessa unidade. Na enfermaria, os sinais vitais (PA, FC, FR e temperatura) foram medidos a intervalos de duas horas no período de vigília e a cada quatro horas durante o período de sono. A freqüência cardíaca e a $\mathrm{SpO}_{2}$ (oxímetro de pulso) foram medidas continuamente durante 24 horas. A sedação foi avaliada por meio de uma escala de seis pontos: 5 - acordado e alerta; 4 - sonolento, mas desperta facilmente; 3 sonolento, mas capaz de abrir os olhos quando chamado várias vezes ou responde a estimulação tátil leve; 2 - sonolento, difícil de ser despertado, precisa ser sacudido; 1 - responde somente a estímulos nocivos; 0 - não responde. A recuperação da anestesia geral foi avaliada pela escala de Aldrete-Kroulik modificada e a qualidade do sono foi avaliada por uma escala de três pontos: 3 - excelente, nenhum despertar; 2 - bom, despertar ocasionais não relacionados com a dor; 1 - ruim, despertar freqüente.

A hipotensão arterial e bradicardia foram definidas como PA sistólica e FC menor que $70 \%$ do valor pré-anestésico. A depressão respiratória foi definida como FR menor que 20 rpm em crianças até 1 ano de idade e menor que 14 rpm para crianças maiores, ou $\mathrm{SpO}_{2}<95 \%$. A infusão foi interrompida por duas horas se a criança parecesse muito sedada (escore $\leq 2$ ), tivesse $\mathrm{SpO}_{2}$ consistentemente menor que $94 \%$ ou depressão respiratória. Se a depressão respiratória fosse intensa (paciente com cianose e bradicardia), os pacientes eram tratados com bolus de naloxona (5 a 10 $\left.\mu \mathrm{g} \cdot \mathrm{kg}^{-1}\right)$ seguida de infusão contínua $\left(5 \mu \mathrm{g} \cdot \mathrm{kg}^{-1} \cdot \mathrm{h}^{-1}\right)$. Foram registrados eventos adversos e comportamentos estranhos. A equipe de anestesia envolvida no estudo permaneceu disponível durante 24 horas para atendimento de eventuais complicações relacionadas com o tratamento analgésico ou sua eventual ineficácia.

Os dados estão apresentados como média \pm DP ou mediana, quando referido. Os escores de sedação e qualidade do sono, dados demográficos, PA, FC, FR, número de doses de tramadol durante 24 horas, e tempo para extubação, para transferência para SRPA ou UTIP, para início da ventilação espontânea e durante o qual a anestesia foi mantida com $\mathrm{N}_{2} \mathrm{O}$ foram comparados entre grupos empregados por meio do teste de Mann-Whitney. As diferenças nas concentrações inspiratórias de isoflurano ao longo do tempo foram analisadas pelo teste de Análise de Variância não-paramétrico para medidas repetidas (teste de Friedman), seguida do pós-teste de Dunn (GraphPad Prism 4). O teste do Qui-quadrado foi empregado para comparar distribuição dos sexos, estado físico ASA e ocorrência de eventos adversos. Para análise estatística $p<0,05$ foi considerado significativo.

\section{RESULTADOS}

Os grupos foram similares com relação aos dados demográficos e duração de intervenções cirúrgicas (Tabela I). Houve uma tendência para pesos e idades menores no Grupo I e esse grupo teve dois pacientes com estado físico ASA III. No entanto, não foi atingida estatística significativa.

As concentrações inspiratórias de isoflurano necessárias para manter a anestesia geral reduziram-se progressivamente e foram muito menores a partir dos 90 e 60 minutos

Tabela I - Dados Demográficos e Duração do Procedimento Cirúrgico

\begin{tabular}{lcc}
\hline & Grupo I $(\mathrm{n}=13)$ & Grupo II $(\mathrm{n}=13)$ \\
\hline $\begin{array}{l}\text { Idade (meses) } \\
\text { Peso (kg) }\end{array}$ & $24,3 \pm 12,2$ & $30,1 \pm 18,5$ \\
Sexo & $9,4 \pm 4,2$ & $11,2 \pm 7,3$ \\
$\quad$ Masculino & 8 & \\
Feminino & 5 & 6 \\
ASA & 5 & 7 \\
I & 6 & 7 \\
II & 2 & 6 \\
III & $148,7 \pm 73$ & $170,6 \pm 82$ \\
$\begin{array}{l}\text { Duração das operações } \\
\text { (min) }\end{array}$ & & \\
\hline
\end{tabular}




\section{ANALGESIA PERIOPERATÓRIA COM INFUSÃO PERIDURAL CONTÍNUA DA COMBINAÇÃO DE MORFINA E CLONIDINA EM CRIANÇAS SUBMETIDAS A PROCEDIMENTOS CIRÚRGICOS ABDOMINAIS}

do início do procedimento cirúrgico nos Grupos I e II, respectivamente, porém não houve diferença entre os grupos (Figura 1). Os tempos de anestesia geral mantido somente com $\mathrm{N}_{2} \mathrm{O} / \mathrm{O}_{2}$ foram similares nos dois grupos (Figura 2). Os tempos para o início da ventilação espontânea, para extubação e para transferência da sala cirúrgica foram similares entre os grupos (Figura 2).

As recuperações da anestesia foram similares nos dois grupos (Figura 3). Marcante sedação foi observada nos pacientes do Grupo II depois de duas horas na SRPA ou UTIP (Figura 4). Na manhã seguinte após a intervenção cirúrgica os pacientes do Grupo II estavam nitidamente mais sedados e tiveram melhor qualidade de sono que os pacientes do Grupo I (Figura 5). O número de doses de tramadol durante 24 horas foi significativamente menor no Grupo II, e somente

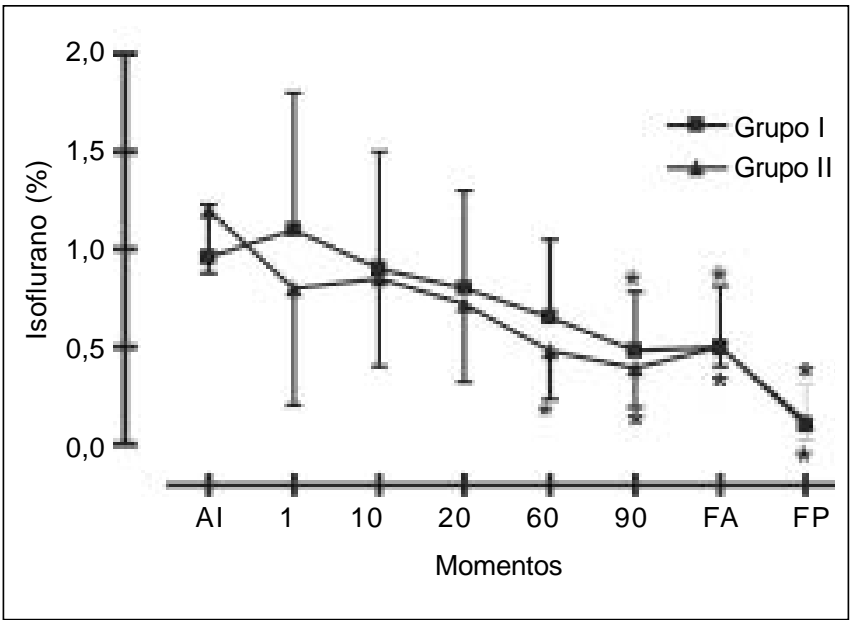

Figura 1 - Concentração Inspiratória de Isoflurano Durante Cirurgias Abdominais em Crianças com Infusão Peridural de Clonidina Mais Morfina. * Estatisticamente significante em relação a $\mathrm{Al}$. $\mathrm{Al}=$ antes da incisão da pele, tempo ( $\mathrm{min}$ ) após a incisão da pele, $\mathrm{FA}=$ fechamento da aponeurose, $\mathrm{FP}=$ fechamento da pele.

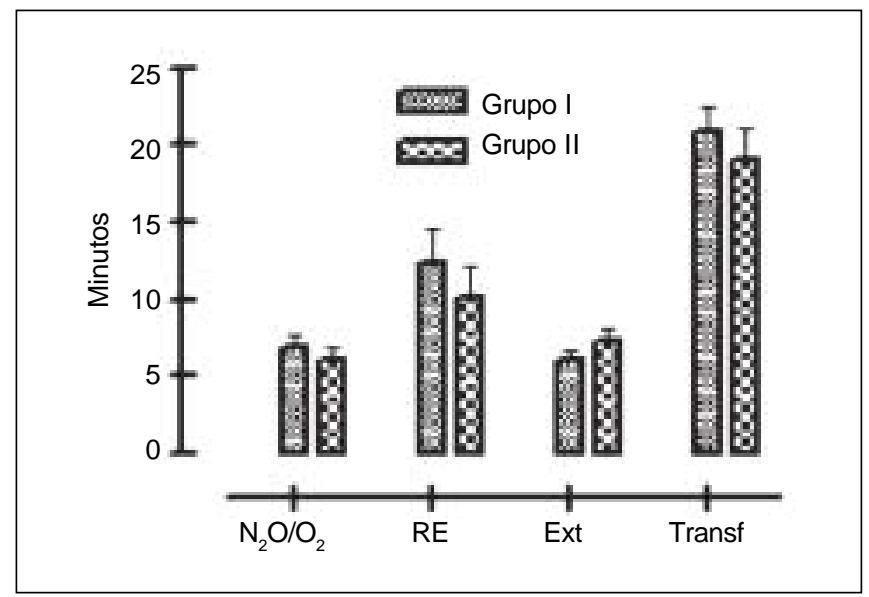

Figura 2 - Tempos de Anestesia Geral somente com Óxido Nitroso $\left(\mathrm{N}_{2} \mathrm{O} / \mathrm{O}_{2}\right)$ e para o Início da Respiração Espontânea (RE) antes do Final da Cirurgia. Tempos para extubação (Ext) e transferência para SRPA ou UTIP (Transf) após o final da cirurgia.

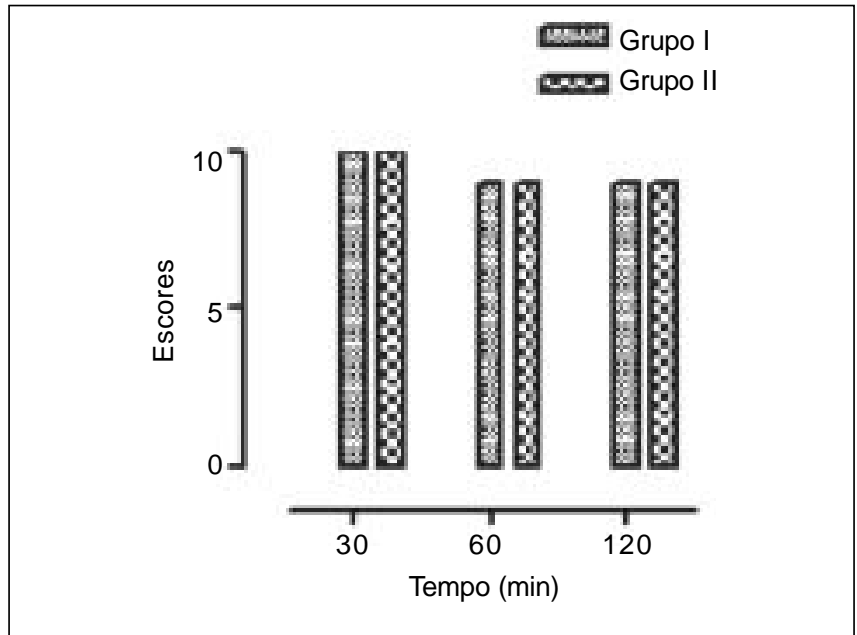

Figura 3 - Recuperação da Anestesia Geral (escala de AldreteKroulik modificada) na SRPA ou UTIP.

Valores representam as medianas.

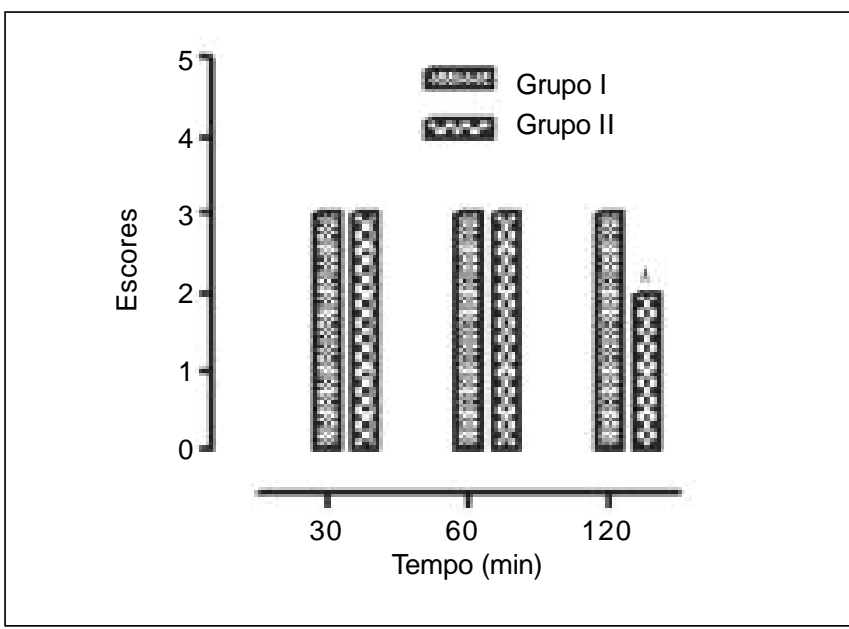

Figura 4 - Sedação na SRPA e UTIP. Valores representam as medianas. *Significativamente diferente.

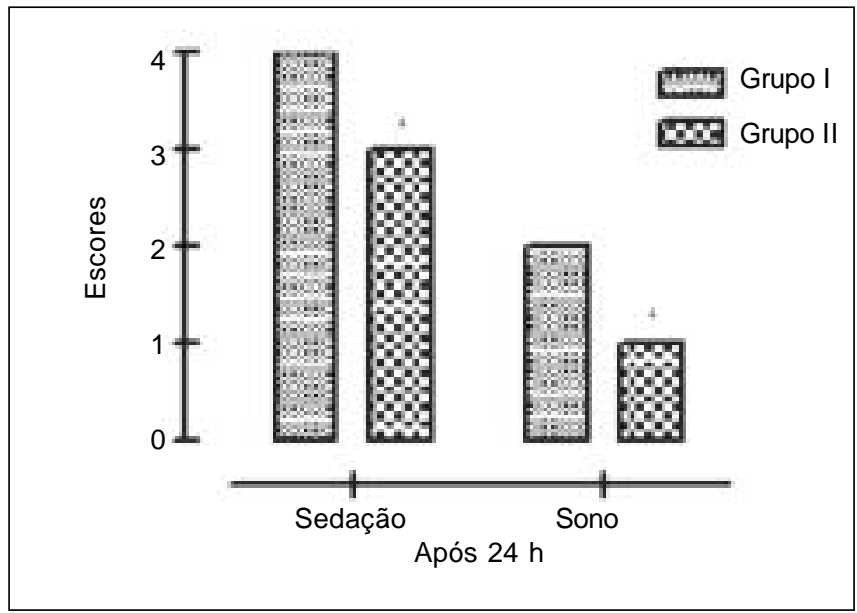

Figura 5 - Avaliação da Qualidade do Sono e Sedação após 24 Horas do Final da Cirurgia. Valores representam as medianas. ${ }^{*}$ Significativamente diferente. 


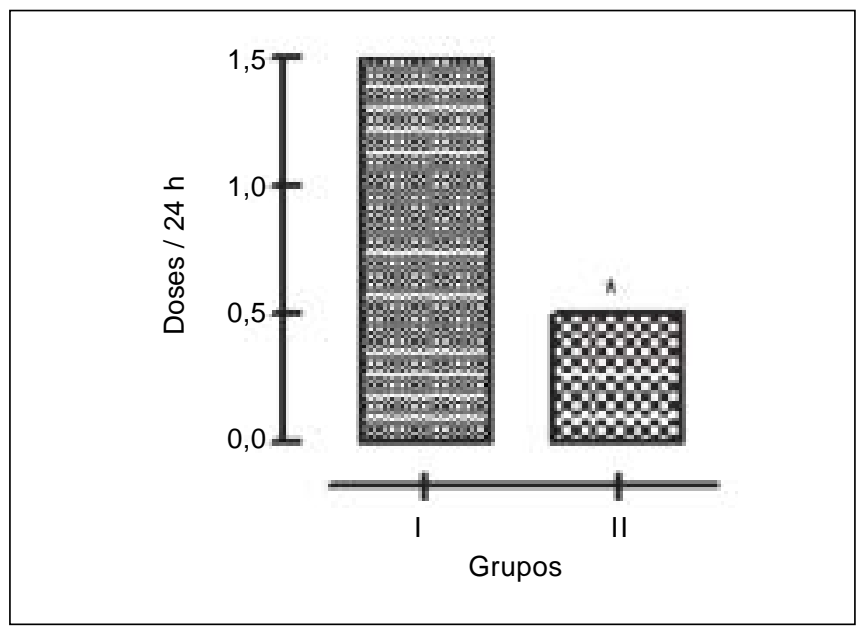

Figura 6 - Número de Doses de Tramadol $\left(1 \mathrm{mg}^{\mathrm{kg}} \mathrm{kg}^{-1}\right)$ durante 24 Horas após o Final da Cirurgia. Valores representam as medianas. *Significativamente diferente.

cinco pacientes $(38,4 \%)$ necessitaram uma ou duas doses de tramadol durante as primeiras 24 horas do pós-operatório (Figura 6).

Três pacientes do Grupo II necessitaram expansão de volemia (realizada com Ringer com lactato, $10 \mathrm{~mL} . \mathrm{kg}^{-1}$ ) para restabelecer a pressão arterial após a operação. Nenhum episódio de bradicardia ou de depressão respiratória foi observado nos dois grupos. Um paciente do Grupo II e dois do Grupo I requereram cateterismo vesical. Prurido foi observado em quatro pacientes do Grupo II. A maioria dos pacientes tolerou bem a presença do tubo nasogástrico, sobretudo os pacientes que receberam ritmo de infusão mais alto. Apenas uma criança do Grupo II teve vômitos.

\section{DISCUSSÃO}

Este estudo demonstrou que a infusão peridural contínua de baixas doses de clonidina e morfina administradas antes do início das intervenções cirúrgicas abdominais de grande porte em crianças com idades entre 4 e 48 meses promoveu analgesia pós-operatória de boa qualidade nas duas doses empregadas. A analgesia intra-operatória, obtida com os dois regimes de infusão peridural, medida pelo consumo de isoflurano, foi demonstrada consistentemente pela sua redução progressiva ao longo da operação. O regime de dose maior (Grupo II) promoveu analgesia de qualidade melhor, confirmada pelo menor consumo de tramadol e analgesia aparentemente completa em $53,8 \%$ dos pacientes, associada à melhor qualidade do sono. O presente estudo também forneceu evidências da segurança da combinação efetiva de morfina associada à clonidina por via peridural em crianças, apesar da sedação prolongada e do risco de hipotensão arterial pós-operatória que respondeu prontamente à reposição volêmica. Não houve depressão respiratória imediata ou tardia ou alta da incidência de vômitos.
As concentrações de isoflurano necessárias para manter a estabilidade cardiovascular intra-operatória foram reduzidas nos dois grupos. A redução da pressão arterial, um indicador do nível de anestesia, não foi acompanhada de redução significativa da freqüência cardíaca. O efeito redutor do consumo de isoflurano foi mantido ao longo da intervenção cirúrgica e na maioria dos pacientes a anestesia geral durante o fechamento da pele pôde ser mantida com $\mathrm{N}_{2} \mathrm{O} / \mathrm{O}_{2}$. Por outro lado, a recuperação imediata da anestesia geral foi similar nos dois grupos. Possivelmente, devido à natureza, intensidade da estimulação cirúrgica e aos efeitos do fentanil, a latência para o efeito poupador de isoflurano em ambos os grupos foi maior que os relatados para a instalação da analgesia após administração peridural de clonidina e morfina em adultos ${ }^{12}$. Entretanto, a latência para redução da necessidade de isoflurano aqui observado coincidiu com o pico de concentração liquórica de ambos os fármacos após administração peridural ${ }^{17}$ e coincidiu também com o tempo entre a administração peridural de clonidina e a máxima redução da pressão arterial observada em estudo prévio ${ }^{7}$ e relatado em dois outros estudos ${ }^{18,19}$. As baixas doses de ataque de clonidina $\left(0,8 \mu \mathrm{g} \cdot \mathrm{kg}^{-1}\right)$ e morfina $\left(8 \mu \mathrm{g} \cdot \mathrm{kg}^{-1}\right)$ foram escolhidas porque se observou hipotensão arterial associada à taquicardia após doses maiores que 1 e $10 \mu \mathrm{g} \cdot \mathrm{kg}^{-1}$, respectivamente, em duas crianças com menos de 6 meses de vida.

A titulação das doses (ou concentrações) de anestésicos gerais segundo as variações hemodinâmicas provocadas pela estimulação cirúrgica é um método comum e aceitável de conduzir a anestesia geral. A estabilidade hemodinâmica, refletida pela redução da necessidade de isoflurano, pode ser o resultado de um efeito anti-hipertensivo específico da combinação de clonidina e morfina por via peridural que mascara a resposta hemodinâmica ao estresse cirúrgico. Assim, os seus efeitos cardiovasculares e analgésicos podem não estar dissociados. A estimulação nociceptiva é um importante determinante da necessidade de anestésicos gerais, e qualquer redução nesse sentido pode ser atribuída a um potente efeito analgésico ${ }^{14}$. Um intenso efeito poupador de anestésicos gerais acarretado pela clonidina ou pela clonidina associada a opióides, independentemente da via de administração, está bem documentado ${ }^{1,12,14-16}$. A impressão dos autores é que a redução na necessidade de isoflurano produzida pela administração peridural contínua deveu-se a efeito analgésico. De fato, apesar dos níveis muito superficiais da anestesia geral administrada (concentração de isoflurano) e completa recuperação da transmissão neuromuscular (TOF > 3 e padrão normal de ventilação espontânea) no final da intervenção cirúrgica, nenhum paciente exibiu sinais de dor (lacrimejamento, salivação ou movimentos grosseiros). Tal efetividade é similar aos efeitos obtidos com o uso rotineiro de bupivacaína a 0,25\%, por via peridural caudal, em crianças. Esse resultado é também consistente com o relato sobre os efeitos poupadores de anestésicos gerais com o uso intra-operatório de clonidina 
por via peridural que foi mais efetiva que o sufentanil ${ }^{13}$ ou mesmo a bupivacaína a $0,25 \%$ pela mesma via, apesar de que comparativamente doses elevadas de clonidina foram empregadas.

Parece evidente que a infusão peridural de baixas doses de clonidina e morfina (Grupo I) promoveu bom controle da dor pós-operatória, e o dobro da dose (Grupo II) promoveu analgesia quase completa para a maioria das crianças submetidas a intervenções cirúrgicas abdominais de grande porte. Tramadol vem sendo empregado em nossa instituição no manuseio de dor pós-operatória em crianças com mais de 3 meses de vida, como alternativa à morfina. As doses cumulativas de analgésicos de resgate (tramadol), um dos parâmetros de eficácia do presente estudo, que depende da intensidade da dor e qualidade do sono, podem representar uma avaliação sensível e objetiva da efetividade do método de analgesia pós-operatória em crianças ${ }^{10}$. Assim, os resultados mostraram uma clara relação dose-efeito entre os dois grupos. O regime de dose maior e a razão morfina/ clonidina para infusão contínua foram obtidos e modificados e partir de um estudo de Motsh e col. ${ }^{8}$ e de um estudo prévio em nossa instituição ${ }^{7}$. Mesmo no regime de doses mais altas (Grupo II), a dose total da morfina administrada em 24 horas $\left(64,6 \mu \mathrm{g} \cdot \mathrm{kg}^{-1}\right)$ é equivalente à faixa de doses seguras mais baixas recomendadas para pediatria ${ }^{6,14,20,21}$, que parecem oferecer baixa probabilidade de depressão respiratória tardia. De fato, nenhuma indicação de efeito depressor respiratório foi detectada nos pacientes dos dois grupos. Esses resultados também são semelhantes aos de De Negri e col. ${ }^{5}$ e Cucchiaro e col. ${ }^{6}$ que descreveram que a infusão contínua peridural de clonidina associada à ropivacaína promove analgesia de boa qualidade em crianças. Coincidentemente, as doses de clonidina consideradas efetivas foram exatamente as mesmas $\left(0,12\right.$ e $\left.0,24 \mu \mathrm{g} \cdot \mathrm{kg}^{-1} \cdot \mathrm{h}^{-1}\right)$ usadas na presente investigação. Até o momento, não há dados clínicos disponíveis a respeito da infusão peridural da combinação de morfina e clonidina em crianças.

A infusão peridural contínua de clonidina, à semelhança do fentanil, produz uma dispersão liquórica cefálica maior que a injeção única e pode ter ação analgésica em todos os segmentos medulares, independentemente da posição do cateter no espaço peridural ${ }^{22}$. Um outro benefício potencial da infusão peridural contínua consiste na analgesia constante de boa qualidade, uma vez que os pacientes são mantidos em um "corredor analgésico" entre a analgesia inadequada e os efeitos adversos e deve ser seguida por picos de concentrações no líquor e no plasma de baixas amplitudes, reduzindo, assim, o risco potencial de efeitos adversos ${ }^{13}$. Exceto pela notória sedação prolongada e três episódios temporários de hipotensão arterial pós-operatória, somente efeitos adversos aceitáveis causados pela morfina peridural, tais como prurido e retenção urinária, ocorreram. A infusão peridural combinada de morfina e clonidina pareceu ser um tratamento analgésico promissor e coerente com o princípio da analgesia multimodal ("analgesia ba- lanceada") advogada por Kehlet ${ }^{23}$. A adição de outros fármacos com mecanismos e locais de ação diferentes (vias diferentes), tais como antiinflamatórios não-hormonais, poderia melhorar o alívio da dor e a segurança. Estudos futuros serão necessários para o estabelecimento de doses precisas da combinação de morfina e clonidina por via peridural que possibilitem a produção de analgesia efetiva com efeitos adversos aceitáveis, por meio da classificação de pacientes por idade, tipo de intervenção cirúrgica, condições clínicas e pontuação do nível de estresse.

O presente estudo demonstrou que dois regimes de dose de infusão contínua peridural da combinação de morfina e clonidina (clonidina $0,12 \mu \mathrm{g} \cdot \mathrm{kg}^{-1} \cdot \mathrm{h}^{-1}$ mais morfina $1,2 \mu \mathrm{g} \cdot \mathrm{kg}^{-1} \cdot \mathrm{h}^{-1}$ e clonidina $0,24 \mu \mathrm{g} \cdot \mathrm{kg}^{-1} \cdot \mathrm{h}^{-1}$ mais morfina $\left.2,4 \mu \mathrm{g} \cdot \mathrm{kg}^{-1} \cdot \mathrm{h}^{-1}\right) \mathrm{du}-$ rante 24 horas no período pós-operatório, precedida de um bolus de clonidina $\left(0,8 \mu \mathrm{g} \cdot \mathrm{kg}^{-1}\right)$ e de morfina $\left(8 \mu \mathrm{g} \cdot \mathrm{kg}^{-1}\right)$, iniciada antes do início cirúrgico, reduziu a necessidade intra-operatória de isoflurano e promoveu analgesia pós-operatória dose-relacionada em crianças de 4 a 48 meses submetidas a procedimento cirúrgico abdominal de grande porte. $\mathrm{O}$ regime de dose maior promoveu analgesia de boa qualidade na maioria das crianças e foi associado à marcante sedação e à ocorrência de hipotensão arterial temporária, sem efeito depressor respiratório.

\section{Perioperative Analgesia with Continuous Epidural Infusion of Morphine Combined with Clonidine in Children Undergoing Abdominal Surgeries}

Jyrson Guilherme Klamt, TSA, M.D.; Magaly Santoni, M.D.; Luis Vicente Garcia, TSA, M.D.; Renato Mestriner Stocche, TSA, M.D.

\section{INTRODUCTION}

The epidural administration of clonidine, a $\alpha_{2}$-adrenergic agonist, produces analgesia ${ }^{1}$ and prolongs the analgesia produced by epidural caudal ropivacaine in children ${ }^{2}$. Since it has a limited action after a bolus administration, continuous infusion is necessary to prolong postoperative analgesia. There are few reports, even in adults, in the literature on the doses of the epidural infusion of clonidine ${ }^{3,4}$. Recently, two reports confirmed the safety and efficacy of the epidural infusion of clonidine associated with small doses of bupivacaine in children ${ }^{5,6}$. A preliminary study ${ }^{7}$ demonstrated that the epidural administration of a bolus of clonidine, associated or not with ropivacaine, followed by the epidural infusion of the same drugs, promoted analgesia of good quality against pain at rest in children undergoing intracavitary abdominal surgeries. However, the safety of the epi- 
dural administration of clonidine is limited by the sedation and hypotension it causes ${ }^{7}$.

Several studies in adults demonstrated the potent interaction of the concomitant epidural administration of morphine and clonidine on the relief of postoperative pain in abdominal surgeries ${ }^{8-12}$.

The administration of small doses of morphine and clonidine reduces, significantly, the risk of severe side effects of epidural morphine (respiratory depression, urinary retention, vomiting, pruritus, and sedation) and epidural clonidine (sedation and hypotension). Besides, clonidine does not intensify the late respiratory depression, the most feared side effect after the epidural administration of morphine. The short duration of action of both drugs makes continuous infusion more adequate for prolonged pain relief after major surgeries when compared with intermittent injections ${ }^{13}$. Despite the proven efficacy of the combination of morphine and clonidine in adults, there are no studies evaluating different regimens of continuous epidural administration of morphine combined with clonidine for pain control in the pediatric population. In addition, the possibility of reduced need of intraoperative general anesthetics could be an added benefit resulting from the combination of morphine and clonidine 1,12,14-16. Thus, the present study evaluated the efficacy of the epidural infusion of low doses of morphine and clonidine in children undergoing major abdominal surgeries.

\section{METHODS}

After approval by the Ethics Committee on Research of the Hospital das Clínicas of the Faculdade de Medicina de Ribeirão Preto - USP, and after parents or legal guardians signed an informed consent, 26 children, ages ranging from 4 to 48 months, physical status ASA I to III, scheduled for major abdominal surgeries, such as Nissen fundoplication, excision of intra-abdominal tumors, splenectomy, or intracavitary urologic surgeries, were enrolled in the study. Patients were electronically allocated, at random (Microsoft Excel, Windows), in two non-blind treatment groups of postoperative analgesia. Both groups received continuous infusion of a mixture of clonidine $\left(1.2 \mu \mathrm{g} \cdot \mathrm{mL}^{-1}\right)$ and morphine $\left(12 \mu \mathrm{g} \cdot \mathrm{mL}^{-1}\right)$ in normal saline for 24 hours via an epidural catheter, preceded by a bolus dose of clonidine $\left(0.8 \mu \mathrm{g} . \mathrm{kg}^{-1}\right)$ and morphine $\left(8 \mu \mathrm{g} \cdot \mathrm{kg}^{-1}\right)$ approximately 5 minutes before the incision. The infusion was administered at 0.1 and $0,2 \mathrm{~mL} \cdot \mathrm{kg}^{-1} \cdot \mathrm{h}^{-1}$ in Groups I and II, respectively. Exclusion criteria included refusal by parents or legal guardians of the epidural anesthesia, neurologic disorders, cardiovascular disorders, or hemodynamic instability.

The children were placed in lateral decubitus and an epidural catheter (20G, Becton-Dickinson) was inserted using the lumbar $\left(L_{1}-L_{5}\right)$ or caudal (sacral hiatus) approach through a Touhy needle (18G, Becton-Dickinson) about $5 \mathrm{~cm}$ into the epidural space. Only children in whom the catheter was ea- sily introduced and met normal resistance to the injection were included in the study.

General anesthesia was induced with midazolam (0.1 mg. $\left.\mathrm{kg}^{-1}\right)$, lidocaine $\left(1.5 \mathrm{mg} \cdot \mathrm{kg}^{-1}\right)$, propofol $\left(3-5 \mathrm{mg} \cdot \mathrm{kg}^{-1}\right)$, fentanyl $(5 \mu \mathrm{g}$. $\left.\mathrm{kg}^{-1}\right)$, dexamethasone $\left(1 \mathrm{mg} \cdot \mathrm{kg}^{-1}\right)$, ondansentron $\left(0.2 \mathrm{mg} \cdot \mathrm{kg}^{-1}\right)$, and pancuronium $\left(0.15 \mathrm{mg} \cdot \mathrm{kg}^{-1}\right)$, and maintained with $\mathrm{N}_{2} \mathrm{O}$ $\left(\mathrm{N}_{2} \mathrm{O} / \mathrm{O}_{2}, 1: 1\right)$ and isoflurane. The inspired concentration of isoflurane was titrated to maintain hemodynamic stability, which was defined as a change in systolic blood pressure and heart rate of no more than $20 \%$ of baseline parameters. Inspired concentrations of isoflurane were recorded immediately before the incision, and 10, 20, 30, 40, 50, 60, and 90 minutes after the incision, and during closure of the aponeurosis and the skin. During the surgery, Ringer's lactate (12-15 mL. kg $\left.{ }^{-1} \cdot \mathrm{h}^{-1}\right)$ and D5W (5 mg. $\left.\mathrm{kg}^{-1} \cdot \mathrm{mm}^{-1}\right)$ were administered intravenously. An orogastric tube was introduced during the surgery for continuous gastric drainage.

After closure of the abdominal wall, the residual neuromuscular blockade was reversed with atropine $\left(25 \mu \mathrm{g} . \mathrm{kg}^{-1}\right)$, and neostigmine $\left(50 \mu \mathrm{g} \cdot \mathrm{kg}^{-1}\right)$ and spontaneous ventilation was allowed if patients maintained $\mathrm{SpO}_{2} \geq 95 \%$ and $\mathrm{P}_{\mathrm{ET}} \mathrm{CO}_{2} \leq$ $55 \mathrm{mmHg}$. The interval between surgery and the beginning of spontaneous ventilation, and the duration of general anesthesia maintained only with $\mathrm{N}_{2} \mathrm{O}$, in minutes, were recorded. The children were extubated when they presented a strong reaction to the tracheal tube, had normal respiratory rate and pattern, $\mathrm{SpO}_{2} \geq 97 \%\left(\mathrm{FiO}_{2}=1.0\right)$, and $\mathrm{P}_{\mathrm{ET}} \mathrm{CO}_{2}<45 \mathrm{mmHg}$, opened their eyes, and moved all four limbs. After the surgery, the children were transferred to the recovery room or to the Pediatric Intensive Care Unit (PICU) (patients younger than 6 months) when they were able to maintain $\mathrm{SpO}_{2} \geq 95 \%$ after five minutes breathing room air and achieved recovery scores (modified Aldrete-Kroulik scale ${ }^{14}$ ) $\geq 7$, and sedation scores (scale from $0-5$ ) $\geq 3$. The length of time necessary for extubation and transference to the recovery room or PICU was recorded.

The nursing staff was oriented to administer intravenous (rescue analgesia) tramadol (1 mg. $\mathrm{kg}^{-1}$, a maximum of $3 \mathrm{mg} \cdot \mathrm{kg}^{-1}$ in a 6-hour period) when they identified pain or discomfort in the patient: non-consolable crying and agitation, complaint of pain, difficulty to cough, and difficulty mobilize in bed due to pain, or presented systolic blood pressure or heart rate 20$30 \%$ greater than preoperative values. Tramadol solutions (1 mg. $\mathrm{mL}^{-1}$ ) were always available for immediate administration, as prescribed by the anesthesia team, and administered according to the evaluation of the nurse responsible for the patient. Parents or guardians were instructed on possible signs of pain/discomfort and stimulated to communicate to the nursing staff if they felt their child was experienced pain and/or discomfort. The quality of postoperative anesthesia was evaluated by the number of doses of tramadol requested in the first 24-postoperative hours, and it was considered the second efficacy parameter in the study. Patients in this study did not receive morphine and/or dypirone during those 24 hours. 


\section{PERIOPERATIVE ANALGESIA WITH CONTINUOUS EPIDURAL INFUSION OF MORPHINE COMBINED WITH CLONIDINE IN CHILDREN UNDERGOING ABDOMINAL SURGERIES}

Sedation, blood pressure (BP), heart rate (HR), recovery from anesthesia (modified Aldrete-Kroulik scale), and respiratory rate (RR) were recorded at 30-minute intervals in the recovery room and PICU up to 120 minutes. Children who remained in the recovery room were discharged to the room 120 minutes after their arrival to this unit. In the room, vital signs (BP, HR, RR, and temperature) were recorded at 2-hour intervals, while patients were awake, and 4-hour intervals when they were sleeping. Heart rate and $\mathrm{SpO}_{2}$ (pulse oximetry) were recorded continuously for 24 hours. Sedation was evaluated using a 6-point scale: 5 - awake and alert; 4 - sleepy, but easily aroused; 3 - sleepy, but opens eyes when called several times or answers to light tactile stimulation; 2 - sleepy, difficult to arouse, has to be shaken; 1 - responds only to pain stimuli; and 0 - no response. Recovery from general anesthesia was evaluated by the modified Aldrete-Kroulik scale, and sleep quality was evaluated by a 3-point scale: 3 - excellent; sleeps without interruption; 2 - good: wakes occasionally, but not due to pain; 1 - poor: awakes frequently.

Hypotension and bradycardia were defined as systolic BP and HR less that $70 \%$ of pre-anesthetic values. Respiratory depression was defined as a RR below $20 \mathrm{bpm}$ in children 1 -year old or younger, and below $14 \mathrm{bpm}$ in older children, or $\mathrm{SpO}_{2}<95 \%$. Infusion was interrupted for 2 hours if the child seemed too sedate (score $\leq 2$ ), $\mathrm{SpO}_{2}$ was consistently below $94 \%$, or developed respiratory depression. In case of severe respiratory depression (patient with cyanosis and bradycardia), patients were treated with a bolus of naloxone ( 5 to $\left.10 \mu \mathrm{g} \cdot \mathrm{kg}^{-1}\right)$ followed by continuous infusion $\left(5 \mu \mathrm{g} \cdot \mathrm{kg} \cdot \mathrm{h}^{-1}\right)$. Adverse events and strange behavior were recorded. The anesthesia team involved in the study remained available for 24 hours in case the child developed any complication related with the analgesic treatment or its eventual lack of efficacy.

Data are presented as mean \pm SD or, when mentioned, median. Sedation and sleep quality scores, demographic data, $\mathrm{BP}, \mathrm{HR}, \mathrm{RR}$, number of doses of tramadol during 24 hours, and time until extubation, to transfer to the recovery room or $\mathrm{PICU}$, to begin spontaneous ventilation, and period anesthesia was maintained with $\mathrm{N}_{2} \mathrm{O}$ were compared in both groups by the Mann-Whitney test. Differences in inspiratory concentration of isoflurane were compared by non-parametric Analysis of Variance for repeated measurements (Friedman test), followed by Dunn's post test (GraphPad Prism 4). The Chi-square test was used to compare the gender distribution, ASA status, and the occurrence of adverse events. A $p<0.05$ was considered significant.

\section{RESULTS}

Both groups were similar regarding the demographic data and duration of surgical interventions (Table I). There was a tendency for lower weight and age in Group I, and this group had two patients with ASA III. However, this difference was not statistically significant.
Table I - Demographic Data and Duration of the Surgery

\begin{tabular}{lcc}
\hline & Group I $(\mathrm{n}=13)$ & Group II $(\mathrm{n}=13)$ \\
\hline Age (months) & $24.3 \pm 12.2$ & $30.1 \pm 18.5$ \\
Weight $(\mathrm{kg})$ & $9.4 \pm 4.2$ & $11.2 \pm 7.3$ \\
Gender & & \\
$\quad$ Male & 5 & 6 \\
Female & 5 & 7 \\
ASA & 5 & 7 \\
I & 6 & 6 \\
II & 2 & 0 \\
III & $148.7 \pm 73$ & $170.6 \pm 82$ \\
Duration of surgeries (min) &
\end{tabular}

Inspiratory concentrations of isoflurane necessary to maintain general anesthesia reduced progressively and were significantly lower at 90 and 60 minutes after the beginning of the procedure in groups I and II, respectively; however, there was no difference between both groups (Figure 1). The period general anesthesia was maintained only with $\mathrm{N}_{2} \mathrm{O} / \mathrm{O}_{2}$ was similar in both groups (Figure 2). The length of time until the beginning of spontaneous ventilation, extubation, and discharge from the operating room were similar in both groups (Figure 2).

Recovery from anesthesia was also similar in both groups (Figure 3). Severe sedation was observed in patients in Group II after 2 hours in the recovery room or PICU (Figure 4). The morning after the surgery, patients in Group II were visibly more sedated and had better sleep quality than patients in

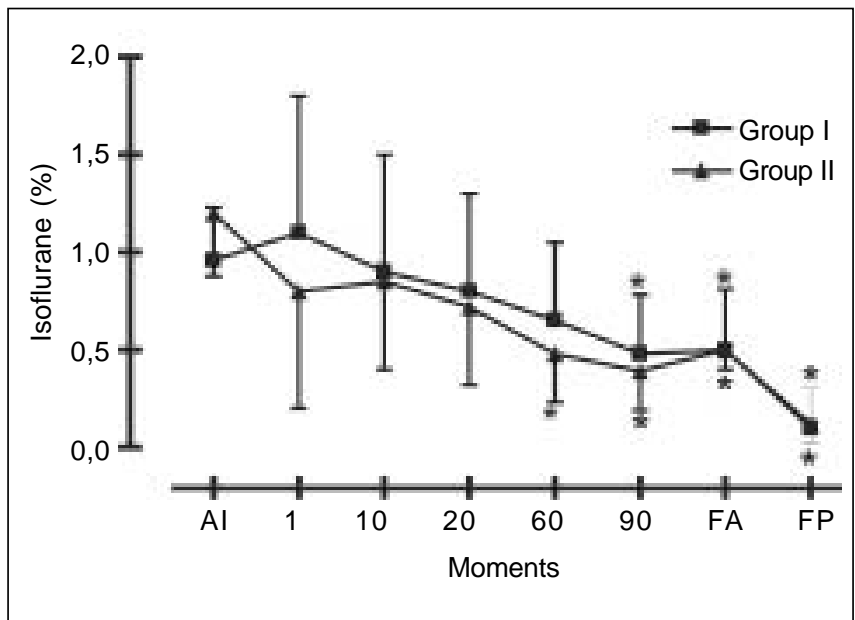

Figure 1 - Inspired Concentration of Isoflurane During Abdomina Surgery in Children with Epidural Infusion of Clonidine and Morphine. * Statistically significant compared to $\mathrm{Al}$. Al= before incision of the skin, time (min) after the incision, $F A=$ closing of aponeurosis, $\mathrm{FP}=$ closing of the skin. 


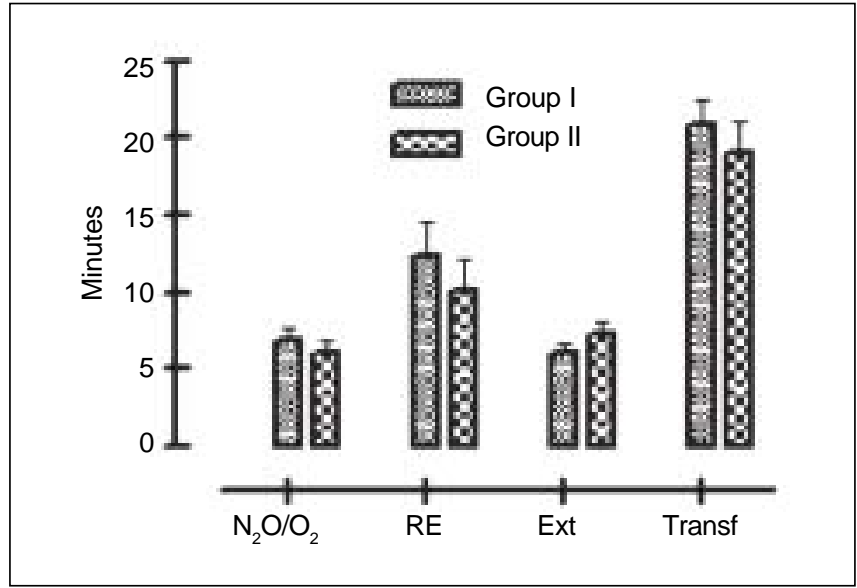

Figure 2 - Period of General Anesthesia with Nitrous Oxide $\left(\mathrm{N}_{2} \mathrm{O} / \mathrm{O}_{2}\right)$ alone and until the Beginning of Spontaneous Breathing (RE) before the End of Surgery. Length of time until extubation (Ext) and transference to the recovery room or PICU (Transf) after the surgery.

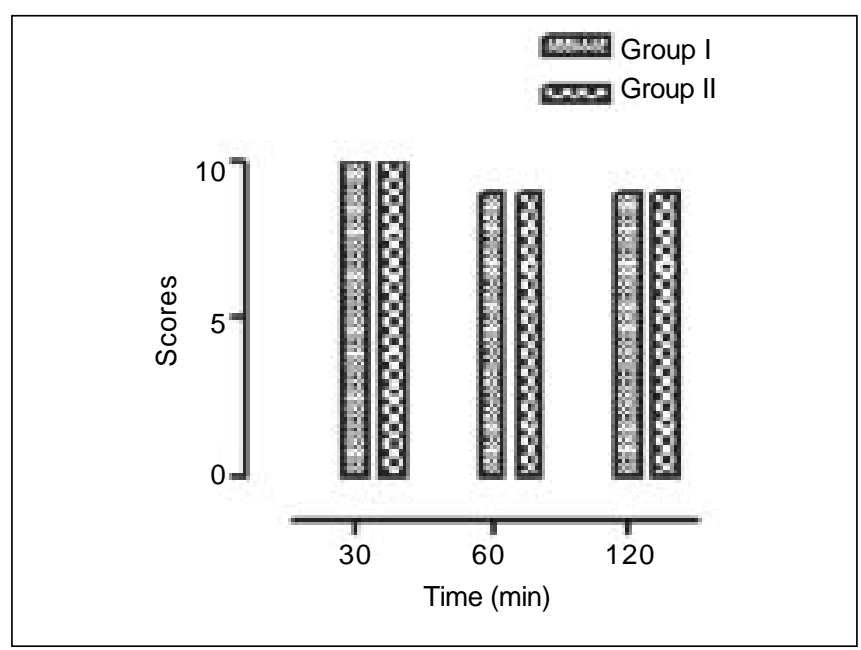

Figure 3 - Recovery from General Anesthesia (modified AldreteKroulik scale) in the Recovery Room or PICU.

Values expressed as median.

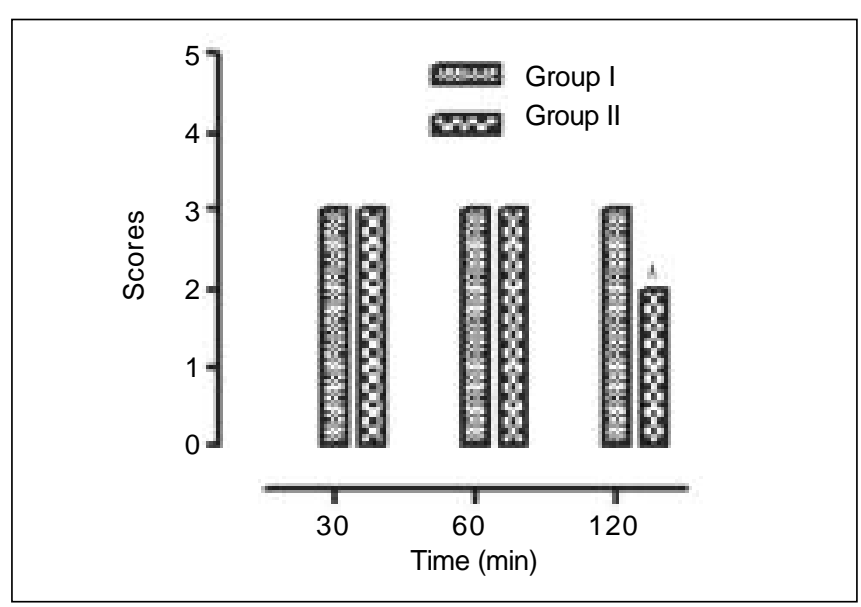

Figure 4 - Sedation in the Recovery Room and PICU. Values expressed as medians. *Statistically significant difference.
Group I (Figure 5). The number of doses of tramadol during 24 hours was significantly lower in Group II, in which only 5 patients $(38.4 \%)$ needed one or two doses of tramadol in the first 24 hours of the postoperative period. (Figure 6).

Three patients in Group II needed volume expansion (with Ringer's lactate, $10 \mathrm{~mL} . \mathrm{kg}^{-1}$ ) to increase their blood pressure after the surgery. Bradycardia and respiratory depression were not observed in both groups. One patient in Group II and 2 in Group I required catheterization of the bladder. Pruritus was observed in 4 patients in Group II. Most patients tolerated well the nasogastric tube, especially patients who received the highest doses. Only one child in Group II developed vomiting.

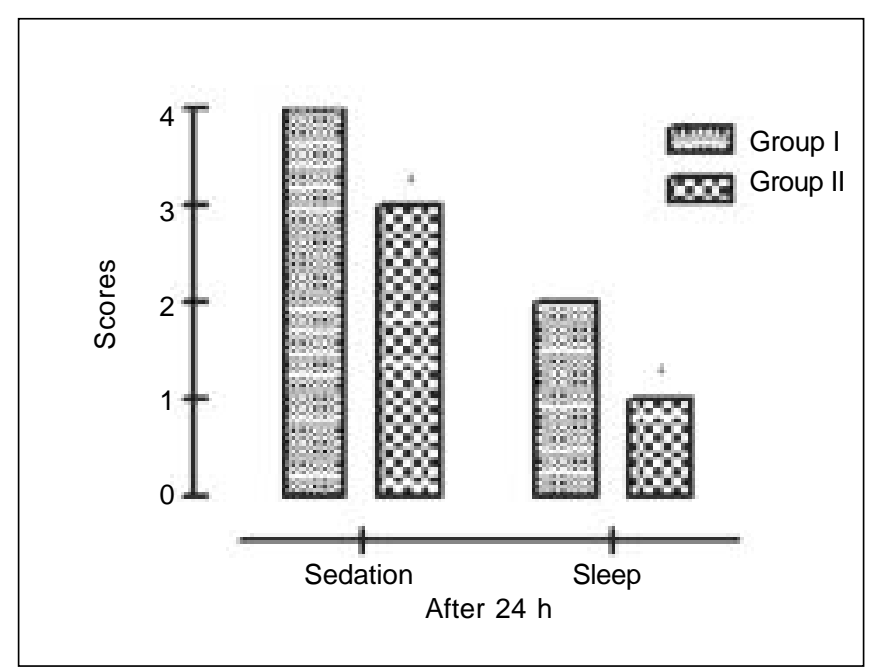

Figure 5 - Evaluation of the Quality of Sleep and Sedation 24 Hours after the Surgery. Values expressed as median.

*Statistically significant difference.

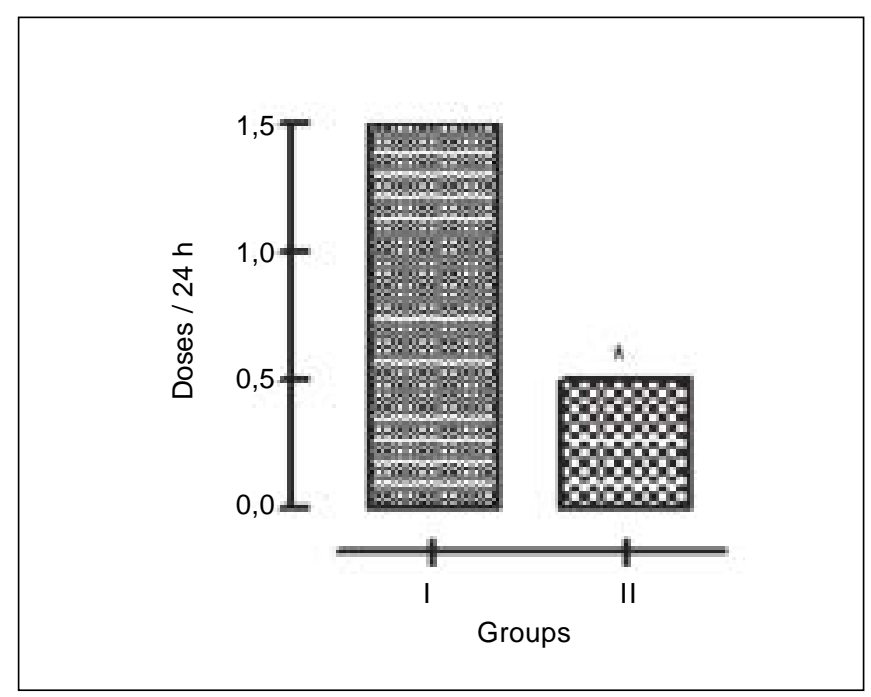

Figure 6 - Number of Doses of Tramadol $\left(1 \mathrm{mg} \cdot \mathrm{kg}^{-1}\right)$ during the First 24 Postoperative Hours. Values expressed as median. *Statistically significant difference. 


\section{DISCUSSION}

This study demonstrated that continuous epidural infusion of low doses of clonidine and morphine, administered before beginning major abdominal surgery, in children aged 4 to 48 months promoted good quality analgesia with both doses. Intraoperative analgesia obtained with both infusion regimens was consistently demonstrated by the progressive reduction in the consumption of isoflurane. The higher dose (Group II) promoted good quality analgesia, confirmed by the reduced consumption of tramadol, apparent analgesia in $53.8 \%$ of the patients, and better sleep quality. The present study also provided evidence of the safety of the combination of epidural morphine and clonidine in children, despite prolonged sedation and the risk of postoperative hypotension, which responded promptly to volume replacement. Immediate or late respiratory depression and high incidence of vomiting were not observed.

Concentrations of isoflurane necessary to maintain intraoperative cardiovascular stability were reduced in both groups. Reduction in blood pressure, an indicator of the level of anesthesia, was not accompanied by a significant reduction in heart rate. The reduction in the consumption of isoflurane was maintained throughout the surgery and, in most patients, general anesthesia during the closure of the skin could be maintained with $\mathrm{N}_{2} \mathrm{O} / \mathrm{O}_{2}$ only. On the other hand, immediate recovery from general anesthesia was similar in both groups. It is possible that, due to the nature, intensity of the surgical stimulation, and the effects of fentanyl, the latency of the isoflurane sparing effect in both groups was greater than those reported in the literature for installation of analgesia after the epidural administration of clonidine and morphine in adults ${ }^{12}$. However, the latency for the reduction in the need of isoflurane observed in this study coincided with the peak concentration in the cerebrospinal fluid of both drugs after epidural administration ${ }^{17}$ and also coincided with the time between the epidural administration of clonidine and the maximal reduction of blood pressure observed in a previous study ${ }^{7}$ and that reported by other two studies ${ }^{18,19}$. Low attack doses of clonidine $\left(0.8 \mu \mathrm{g} . \mathrm{kg}^{-1}\right)$ and morphine $\left(8 \mu \mathrm{g} . \mathrm{kg}^{-1}\right)$ were chosen because hypotension associated with tachycardia was observed after doses higher then 1 and $10 \mu \mathrm{g} \cdot \mathrm{kg}^{-1}$, respectively, in children younger than 6 months. Titration of the doses (or concentrations) of general anesthetics according to hemodynamic changes provoked by the surgical stimulation is a common and acceptable method of managing general anesthesia. The hemodynamic stability, reflected by the reduction in the need of isoflurane, could have resulted by a specific anti-hypertensive effect of the combination of epidural clonidine-morphine that masked the hemodynamic response to the surgical stress. Thus, it is possible that its cardiovascular and analgesic effects might not be dissociated. The nociceptive stimulation is an important determinant of the need of general anesthetics, and any reduction to that effect can be attributed to a potent analgesic effect ${ }^{14}$. A strong general anesthetics-sparing effect of clonidine, or clonidine associated with opioids, regardless of the route of administration, is well documented ${ }^{1,12,14-16}$. The impression of the authors is that the reduction in the need of isoflurane produced by the continuous epidural administration was a result of the analgesic effect. In fact, despite the very superficial levels of the general anesthesia administered (concentration of isoflurane) and complete recovery of the neuromuscular transmission (TOF $>3$ and a normal pattern of spontaneous ventilation) at the end of the surgery, patients showed no signs of pain (tearing, salivation, or rude movements). Such effectiveness is similar to the effects obtained with the routine use of epidural/caudal $0.25 \%$ bupivacaine in children. This result is also consistent with the report that the general anesthetics-sparing effects of the intraoperative administration of epidural clonidine was more effective than sufentanil ${ }^{13}$, or even $0.25 \%$ bupivacaine using the same route, although with relatively elevated doses of clonidine.

It seems evident that the epidural infusion of low doses of clonidine and morphine (Group I) promoted good control of postoperative pain, and doubled the dose (Group II) promoted almost complete analgesia for most children undergoing major abdominal surgeries. Tramadol has been used in our institution for the management of postoperative pain in children older than 3 months as an alternative to morphine. The cumulative doses of rescue analgesics (tramadol), one of the parameters of efficacy used in this study, which depends on the severity of the pain and sleep quality can be a sensitive and objective evaluation of the effectiveness of the method used to control postoperative pain in children ${ }^{10}$. Thus, the results demonstrated a clear dose-effect relationship in both groups. The regimen with greater dose and the ratio morphine/clonidine for continuous infusion were obtained, and modified, from a study by Motsch et al. ${ }^{8}$ and from a previous study from our institution ${ }^{7}$. Even in the higher-dose regimen (Group II) the total dose of morphine in 24 hours $\left(64.4 \mu \mathrm{g} . \mathrm{kg}^{-1}\right)$ is equivalent to the lower range of safety recommended for the pediatric population ${ }^{6,14,20,21}$ that seem to have low probability of late respiratory depression. In fact, we did not detect any indication of respiratory depression in both groups. These results are also similar to those of De Negri et al. ${ }^{5}$ and Cecchiaro et al. ${ }^{6}$, who reported that the continuous epidural infusion of clonidine associated with ropivacaine promoted analgesia of good quality in children. Coincidently, the doses of clonidine considered effective were exactly the same $(0.12$ and $\left.0.24 \mu \mathrm{g} \cdot \mathrm{kg}^{-1} \cdot \mathrm{h}^{-1}\right)$ used in the present study. There are no current data available on the epidural infusion of the combination of morphine and clonidine in children.

Similar to fentanyl, the continuous epidural infusion of clonidine produces greater cephalad dispersion in the cerebrospinal fluid than a single dose and, for this reason, it can have analgesic effects in all segments of the spinal cord, regardless of the position of the catheter in the epidural space ${ }^{22}$. Another potential benefit of continuous epidural infusion is the 
constant good quality analgesia since patients are maintained in a "analgesic corridor" between inadequate analgesia and adverse effects, and it should be accompanied by peaks of low amplitude of the cerebrospinal fluid and plasma concentration, therefore reducing the potential risk of adverse effects ${ }^{13}$. Except for the prolonged sedation and three temporary episodes of postoperative hypotension, some acceptable side effects caused by epidural morphine, such as pruritus and urinary retention, were detected. Continuous epidural infusion of morphine and clonidine seems to be a promising analgesic treatment, coherent with the principle of multimodal analgesia ("balanced analgesia") advocated by Kehlet ${ }^{23}$. Addition of drugs with different mechanisms and sites of action (different pathways), such as non-steroidal anti-inflammatories, could improve pain relief and safety. Further studies are needed to establish the precise doses of the combination of epidural morphine and clonidine that produce effective analgesia with acceptable side effects, by classifying patients by age, type of surgery, clinical conditions, and scores of the stress level.

The present study demonstrated that two regimens of continuous epidural infusion of the combination of morphine and clonidine (clonidine, $0.12 \mu \mathrm{g} \cdot \mathrm{kg}^{-1} \cdot \mathrm{h}^{-1}$, plus morphine, 1.2 $\mu \mathrm{g} \cdot \mathrm{kg}^{-1} \cdot \mathrm{h}^{-1}$ and clonidine $0.24 \mu \mathrm{g} \cdot \mathrm{kg}^{-1} \cdot \mathrm{h}^{-1}$, plus morphine, 2.4 $\left.\mu \mathrm{g} \cdot \mathrm{kg}^{-1} \cdot \mathrm{h}^{-1}\right)$ in the first 24 postoperative hours, preceded by a bolus of clonidine $\left(0.8 \mu \mathrm{g} \cdot \mathrm{kg}^{-1}\right)$, and morphine $\left(8 \mu \mathrm{g} \cdot \mathrm{kg}^{-1}\right)$ before the surgery, reduced the intraoperative need of isoflurane and promoted postoperative dose-related analgesia in children ages 4 months to 48 months undergoing major abdominal surgery. Higher doses of analgesics promoted good quality analgesia in most children, and were associated with marked sedation and the occurrence of temporary hypotension without respiratory depression.

\section{REFERÊNCIAS - REFERENCES}

01. De Kock M, Crochet B, Morimont C et al. - Intravenous or epidural clonidine for intra- and postoperative analgesia. Anesthesiology, 1993;79:525-531.

02. De Negri $P$, Ivani $G$, Visconti $C$ et al. - How to prolong postoperative analgesia after caudal anaesthesia with ropivacaine in children: S-ketamine versus clonidine. Paediatr Anaesth, 2001;11:679-683.

03. De Kock M, Wiederkher P, Laghmiche A et al. - Epidural clonidine used as the sole analgesic agent during and after abdominal surgery. A dose-response study. Anesthesiology, 1997;86: 285-292.

04. De Kock M, Gautier P, Pavlopoulou A et al. - Epidural clonidine or bupivacaine as the sole analgesic agent during and after abdominal surgery: a comparative study. Anesthesiology, 1999; 90:1354-1362.

05. De Negri P, Ivani G, Visconti C et al. - The dose-response relationship of clonidine added to a postoperative continuous infusion of ropivacaine in children. Anesth Analg, 2001;93:71-76.

06. Cucchiaro G, Dagher C, Baujard C et al. - Side-effects of postoperative epidural analgesia in children: a randomized study comparing morphine and clonidine. Paediatr Anaesth, 2003;13: 318-323.
07. Klamt JG, Garcia LV, Stocche RM et al. - Epidural infusion of clonidine or clonidine plus ropivacaine for postoperative analgesia in children undergoing major abdominal surgery. J Clin Anesth, 2003;15:510-514.

08. Motsh J, Gräber E, Ludwig K - Addition of clonidine enhances postoperative analgesia from epidural morphine: a double-blind study. Anesthesiology, 1990;73:1067-1073.

09. Rostaing S, Bonnet F, Levron JC et al. - Effect of epidural clonidine on analgesia and pharmacokinetics of epidural fentanyl in postoperative patients. Anesthesiology, 1991;75:420-425.

10. Anzai Y, Nishikawa T - Thoracic epidural clonidine and morphine for postoperative pain relief. Can J Anaesth, 1995;42:292-297.

11. Rockemann MG, Seeling W, Brinkmann A et al. - Analgesic and hemodynamic effects of epidural clonidine, clonidine/morphine, and morphine after pancreatic surgery. A double-blind study. Anesth Analg, 1995;80:869-874.

12. Vercauteren M, Lauwers E, Meert T et al. - Comparison of epidural sufentanil plus clonidine with sufentanil alone for postoperative pain relief. Anaesthesia, 1990;45:531-534.

13. Norton NS - Prevention and control of pain in children. $\mathrm{Br} J$ Anaesth, 1999;83:118-129.

14. De Kock M, Famenne F, Deckers G et al. - Epidural clonidine or sufentanil for intraoperative and postoperative analgesia. Anesth Analg, 1995;81:1154-1162.

15. Nishina K, Mikawa K, Maekawa N et al. - The efficacy of clonidine for reducing perioperative haemodynamic changes and volatile anaesthetic requirements in children. Acta Anaesthesiol Scand, 1996;40:746-751.

16. Murga G, Samso E, Valles $\mathrm{J}$ et al. - The effect of clonidine on intra-operative requirements of fentanyl during combined epidural/general anaesthesia. Anaesthesia, 1994;49:999-1002.

17. Glynn CJ, Jamous MA, Teddy PJ - Cerebrospinal fluid kinetics of epidural clonidine in man. Pain, 1992;49:361-367.

18. Motsch J, Böttiger BW, Bach A et al. - Caudal clonidine and bupivacaine for combined epidural and general anaesthesia. Acta Anaesthesiol Scand, 1997;41:877-883.

19. Jamali S, Monin S, Begon C et al. - Clonidine in pediatric caudal anesthesia. Anesth Analg, 1994;78:663-666.

20. Krane EJ, Tyler DC, Jacobsen LE - The dose response of caudal morphine in children. Anesthesiology, 1989;71:48-52.

21. Valley RD, Bailey AG - Caudal morphine for postoperative analgesia in infants and children: a report of 138 cases. Anesth Analg, 1991;72:120-124.

22. Curatolo M, Peterson-Felix S, Arendt-Nielsen $L$ et al. - Epidural epinephrine and clonidine segmental analgesia and effects on different pain modalities. Anesthesiology, 1997;87:785-794.

23. Kehlet $\mathrm{H}-$ Surgical stress: the role of pain and analgesia. $\mathrm{Br} \mathrm{J}$ Anaesth, 1989;63:189-195.

\section{RESUMEN}

Klamt JG, Santoni M, Garcia LV, Stocche RM - Analgesia Perioperatoria con Infusión Peridural Continua de la Combinación de Morfina y Clonidina en Niños Sometidos a Procedimientos Quirúrgicos Abdominales.

JUSTIFICATIVA Y OBJETIVOS: El presente estudio fue desarrollado para evaluar el efecto analgésico de la combinación de morfina y clonidina administrada por vía peridural, cuya eficacia fue demostrada en pacientes adultos, sobre el consumo de isoflurano y el consumo de medicación analgésica en el período postoperatorio en niños.

MÉTODO: Veinte y seis niños destinados a operaciones intra-abdominales fueron ubicados aleatoriamente en de los grupos. Los de 
los grupos recibieron, por vía peridural, bolus de morfina (8 $\left.\mu \mathrm{g} \cdot \mathrm{kg}^{-1}\right)$ $y$ de clonidina $\left(0,8 \mu \mathrm{g} \cdot \mathrm{kg}^{-1}\right)$ antes del inicio de la intervención quirúrgica, seguidos de infusión continua de clonidina $\left(0,12 \mu \mathrm{g} \cdot \mathrm{kg}^{-1} \cdot \mathrm{h}^{-1}\right)$ más morfina $\left(1,2 \mu \mathrm{g} \cdot \mathrm{kg}^{-1} \cdot \mathrm{h}^{-1}\right)$ en el Grupo I y el doble de esa dosis en el Grupo II, durante 24 horas. Fueron medidas las concentraciones inspiratorias de isoflurano durante la operación y el número de dosis $\left(1 \mathrm{mg}^{\mathrm{kg}} \mathrm{H}^{-1}\right)$ de tramadol durante 24 horas en el postoperatorio.

RESULTADOS: Las concentraciones de isoflurano fueron significativamente menores con relación a los valores observados antes de la incisión quirúrgica después 60 y 90 minutos en los grupos II $y \mathrm{l}$, respectivamente, sin embargo no hubo diferencia entre los de los grupos. El consumo de tramadol fue significativamente menor en el grupo 2, siendo que 7 (53,8\%) no necesitaron analgesia de rescate, sin embargo fueron observadas mayor sedación e hipotensión arterial. No fue observada depresión respiratoria en los de los grupos.

CONCLUSIÓN: La infusión peridural de la combinación de bajas dosis de clonidina y morfina promovieron reducción de la necesidad de isoflurano en el período intraoperatorio y analgesia postoperatoria de buena calidad. 